\title{
Functional mechanisms of drought tolerance in subtropical maize (Zea mays L.) identified using genome-wide association mapping
}

Nepolean Thirunavukkarasu ${ }^{*}$, Firoz Hossain ${ }^{1}$, Kanika Arora ${ }^{1}$, Rinku Sharma ${ }^{1}$, Kaliyugam Shiriga ${ }^{1}$, Swati Mittal ${ }^{1}$, Sweta Mohan', Pottekatt Mohanlal Namratha', Sreelatha Dogga², Tikka Shobha Rani², Sumalini Katragadda ${ }^{2}$, Abhishek Rathore ${ }^{4}$, Trushar Shah ${ }^{4}$, Trilochan Mohapatra ${ }^{5}$ and Hari Shankar Gupta ${ }^{1}$

\begin{abstract}
Background: Earlier studies were focused on the genetics of temperate and tropical maize under drought. We identified genetic loci and their association with functional mechanisms in 240 accessions of subtropical maize using a high-density marker set under water stress.

Results: Out of 61 significant SNPs (11 were false-discovery-rate-corrected associations), identified across agronomic traits, models, and locations by subjecting the accessions to water stress at flowering stage, $48 \%$ were associated with drought-tolerant genes. Maize gene models revealed that SNPs mapped for agronomic traits were in fact associated with number of functional traits as follows: stomatal closure, 28; flowering, 15; root development, 5; detoxification, 4; and reduced water potential, 2. Interactions of these SNPS through the functional traits could lead to drought tolerance. The SNPs associated with ABA-dependent signalling pathways played a major role in the plant's response to stress by regulating a series of functions including flowering, root development, auxin metabolism, guard cell functions, and scavenging reactive oxygen species (ROS). ABA signalling genes regulate flowering through epigenetic changes in stress-responsive genes. ROS generated by ABA signalling are reduced by the interplay between ethylene, $A B A$, and detoxification signalling transductions. Integration of ABA-signalling genes with auxin-inducible genes regulates root development which in turn, maintains the water balance by regulating electrochemical gradient in plant.

Conclusions: Several genes are directly or indirectly involved in the functioning of agronomic traits related to water stress. Genes involved in these crucial biological functions interacted significantly in order to maintain the primary as well as exclusive functions related to coping with water stress. SNPs associated with drought-tolerant genes involved in strategic biological functions will be useful to understand the mechanisms of drought tolerance in subtropical maize.
\end{abstract}

Keywords: Genome-wide SNPs, Association mapping, Functional mechanisms, Candidate SNPs, Water stress, Drought tolerance, Maize

\footnotetext{
* Correspondence: tnepolean@gmail.com

'Division of Genetics, Indian Agricultural Research Institute, Pusa, New Delhi

110012, India

Full list of author information is available at the end of the article
}

\section{Biomed Central}

(c) 2014 Thirunavukkarasu et al.; licensee BioMed Central. This is an Open Access article distributed under the terms of the Creative Commons Attribution License (http://creativecommons.org/licenses/by/4.0), which permits unrestricted use, distribution, and reproduction in any medium, provided the original work is properly credited. The Creative Commons Public Domain Dedication waiver (http://creativecommons.org/publicdomain/zero/1.0/) applies to the data made available in this article, unless otherwise stated. 


\section{Background}

Drought at the flowering stage produces infertile pollen in maize (Zea mays L.) [1], resulting in substantially lower yield. A shorter anthesis-to-silking interval (ASI) was observed under drought in a lowland tropical maize population [2], and the interval was considered to be an indirect selection criterion for grain yield [3]. Grain yield, a complex trait dependent on several environmental factors [4], was increased through genetic modifications despite stress [5].

Several researchers have identified drought-related genes in different tissues that regulate molecular and physiological responses under stress [6-8]. In the ears and silks of maize, some genomic regions that control the levels of abscisic acid (ABA) and sugar showed the signal transduction involved in stress-related pathways and regulated kernel size and productivity of the plant under drought [6]. Different tissues showed the expression of an NAC-transcription-factor-encoding gene [9], which was observed to be strongly associated with the relative ear position, 100-kernel weight, and flowering time across the experimental maize population [10]. Some drought response has also been recorded recently in reproductive and leaf meristem tissues in maize [7].

Furthermore, a high-resolution association map created using a high-density marker set has the potential to unravel stress-associated genetic variability in a genome [10]. Considerable efforts have been made to understand the association of SNPs with different phenotypic traits in maize [10-12], and strong SNP associations have been identified for flowering time [13], kernel shape [14], 100-kernel weight [14], and kernel quality [15]. Moreover, target genes for crop improvement have been successfully identified using genome-wide association (GWA) mapping in maize $[6,10,12]$.

In this study, we assembled a GWA mapping panel using elite subtropical maize genotypes and phenotyped it under water stress (WS) at flowering time at multiple locations to identify the SNPs associated with key agronomic traits. The association of genetic loci with the agronomic traits and their recurrence level across different locations and models were examined, to identify the SNPs significantly associated with the candidate genes that interact to confer tolerance to WS.

\section{Results}

\section{Phenotypic data}

An association mapping panel of 240 subtropical maize lines was subjected to WS and the performance of the stressed maize lines was compared with that of the lines grown under a well-watered (WW) environment. The panel was phenotyped for anthesis-to-silking interval (ASI), ear length (EL), ear girth (EG), kernels per row $(\mathrm{KR})$, the number of kernel rows (KRN), 100-kernel weight (HKW), and grain yield (GY) at three locations: Indian Agriculture Research Institute (IARI), New Delhi; Acharya N G Ranga Agricultural University (ANGRAU), Hyderabad; and Agricultural Research Station (ARS), Karimnagar for two years. The overall performance of the hybrids under WS was significantly impaired [Additional file 1: Table S1]. The phenotypic correlation coefficient showed that GY and the traits contributing to it were positively and significantly correlated with each other, and ASI was negatively but significantly correlated with other agronomic traits [Additional file 2: Table S2].

\section{Genome-wide association mapping Genome-wide association models}

We detected GWA signals from GenABEL and Genome Association and Prediction Integrated Tool (GAPIT) models. Model fitness varied over the data sets (traits + models + locations) and an example from GenABEL is given below. For ASI-Hyderabad, a higher model $\left(\mathrm{Q}_{10}+\right.$ $\mathrm{K}+$ Admixture) from GenABEL showed greater fitness than the lower hierarchy models $\left(\mathrm{K}\right.$ and $\left.\mathrm{Q}_{10}+\mathrm{K}\right)$ did. The genomic inflation factor $(\lambda)$ was 1.04 in the latter model but was reduced to 1.02 when admixture was added as a covariate (Figure 1a); $\mathrm{Q}_{10}+\mathrm{K}$ and $\mathrm{Q}_{10}+\mathrm{K}+$ Admixture from GenABEL had equal effect on correcting cryptic relationship for ASI-Karimnagar (Figure 1b); and all the models proved equally good for GY-Hyderabad (Figure 1c).

\section{Comparison of GWA signals: WW and WS}

Of 61 SNPs strongly $\left(P<5.8 \times 10^{-4}\right)$ associated with WS, 11 were false-discovery-rate (FDR)-corrected associations and the rest (50) were consistent across the data sets (traits + models + locations). The maximum number (16) of association signals were identified on chromosome 5 followed by those on chromosome 3 (15), whereas chromosomes 2 and 8 shared the least number of significant associations (2) across traits measured under WS.

Genome-wide analysis was also assessed for the WW data sets, which mapped 70 significant associations $\left(P<5.8 \times 10^{-4}\right)$ including 9 FDR-corrected associations. The maximum number of GWA signals for WW was contributed by the SNPs mapped on chromosome 10 (15) followed by those on chromosomes 1 (12) and 7 (10), whereas the minimum number (2) of GWA signals was shared by the SNPs mapped on chromosome 8 . The recurrence of these signals across all data sets (traits + models + locations) varied with those observed in WS. Also the stringency of these results was observed through the number of significantly associated SNPs for EG trait being 17 in WS and 24 in WW. But, for traits ASI, GY, and $K R$, the number of GWA signals in WW were equivalent to that in WS. SNPs significantly associated in both conditions were mapped near both drought tolerant genes 


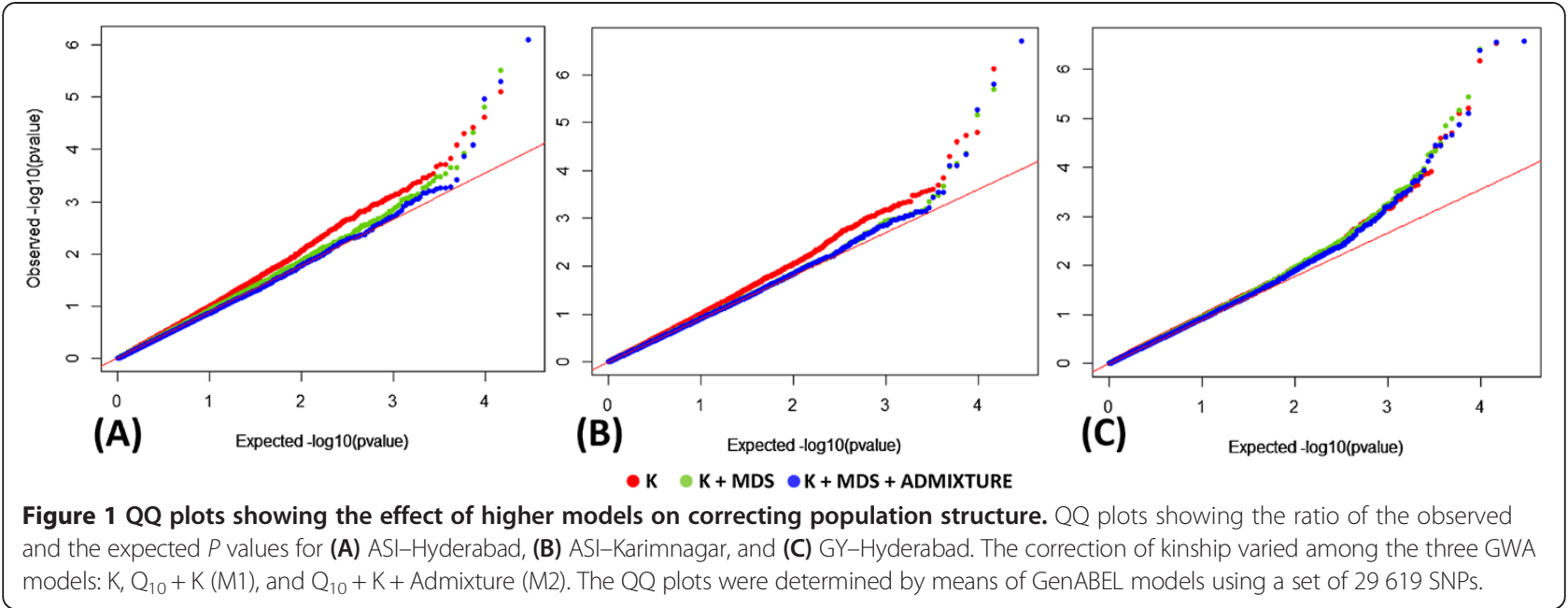

and regulatory genes having their importance in both stressed and normal environment-PZE-107021672 and PZE-107021673 for GY; PZE-101135368 for ASI and KR; PZE-107110985 for ASI, GY, and KR. However rest of the SNPs were also significantly associated in WW and WS but were specific to environmental locations.

\section{GWA signals: WS}

On chromosome 1, a significant signal (PZE-101135368) associated with WS-ASI was located $12 \mathrm{~kb}$ from the nuclear factor-YA transcription factor (NF-YA) [Additional file 3: Table S3]. This SNP also showed an FDR-corrected $P$ value of $5.4 \times 10^{-9}$ for WS-ASI and was repeated $75 \%$ of the times across all data sets (traits + models + locations) under WS (Figure 2). For ASI-New Delhi, PZE-101135368 was significantly associated (at $5 \%$ FDR) with a stronger $P$ value of $5.4 \times 10^{-9}$ for WS [Additional file 3: Table S3] than that for the nonsignificant signal observed under WW $\left(P=1.4 \times 10^{-4}\right)$ [Additional file 4: Figure S1]. In addition, this locus was co-localized with a quantitative trait locus (QTL) earlier mapped for ASI by Almeida et al [16]. Furthermore, significant associations for WS also comprised DnaJ-49like chaperone protein and a domain of unknown function (231) that were co-localized with two other QTLs for ASI on chromosome 1 under WS [16].

Two FDR-corrected associations for WS-ASI, one on chromosome 7 (PZE-107110985) and the other on chromosome 10 (PZE-110016300), were found near a gene encoding a basic helix-loop-helix (bHLH) transcription factor [Additional file 3: Table S3]. The first locus identified at $92 \mathrm{~kb}$ from bHLH was also significantly associated with WS-GY, -EG, - HKW, -EL, -KRN, and -KR at specific locations [Additional file 3: Table S3]. The second locus, mapped at $118 \mathrm{~kb}$ from bHLH, also showed strong associations with WS-HKW, -KRN, and -KR [Additional file 3: Table S3] and significant associations for ASI across all locations and mean data (Figures 2 and $3 \mathrm{a}$ ), while the rest of the traits were confined to specific locations (Figure 2).

SNP PZE-103149505 mapped on chromosome 3, indicated strong GWA signals for WS-ASI across multiple locations (Figure 2). These signals showed a stronger and significant association under WS than that for WW, in which the $P$ values were less significant and less repeatable. This SNP was mapped $30 \mathrm{~kb}$ from a stress-related gene encoding NADP-malic enzyme [Additional file 3: Table S3].

SNPs mapped on chromosome 5 showed five FDRcorrected association signals for WS-GY [Additional file 3: Table S3]: three SNPs (PZE-105073248, PZE-105073274, and PZE-105073275) were consistent across all locations, whereas two (PZE-105073295 and PZE-105076764) were specific to the locations and models (Figure $3 \mathrm{~b}$ ). Of these five SNPs, three (PZE-105073248, PZE-105073275, and PZE-105073274) were clustered in a $131 \mathrm{~kb}$ region, which enclosed genes encoding MYB-related (MYB) transcription factor and squamous promoter binding protein (SBP) transcription factor [Additional file 3: Table S3]. This cluster was identified $65 \%$ of the times across all WS data sets, including GY (Figure 2), and showed strong associations with WS-GY that were more recurrent across locations and models than those in the WW data sets. In addition to this, two FDR-corrected SNPs (PZE-107021673 and PZE-107110985) on chromosome 7 were also strongly associated with WS-GY. The first SNP was mapped nearer to the glycerol-3-phosphate dehydrogenase gene and an ethylene responsive factor (ERF) transcription factor, and the second was close to the bHLH encoding gene [Additional file 3: Table S3]. Another SNP on chromosome 9, $16 \mathrm{~kb}$ from the MYB, was also associated with WS-GY [Additional file 3: Table S3]. All SNPs on chromosomes 7 and 9 also had a significant association with WS-EG [Additional file 3: Table S3]. 


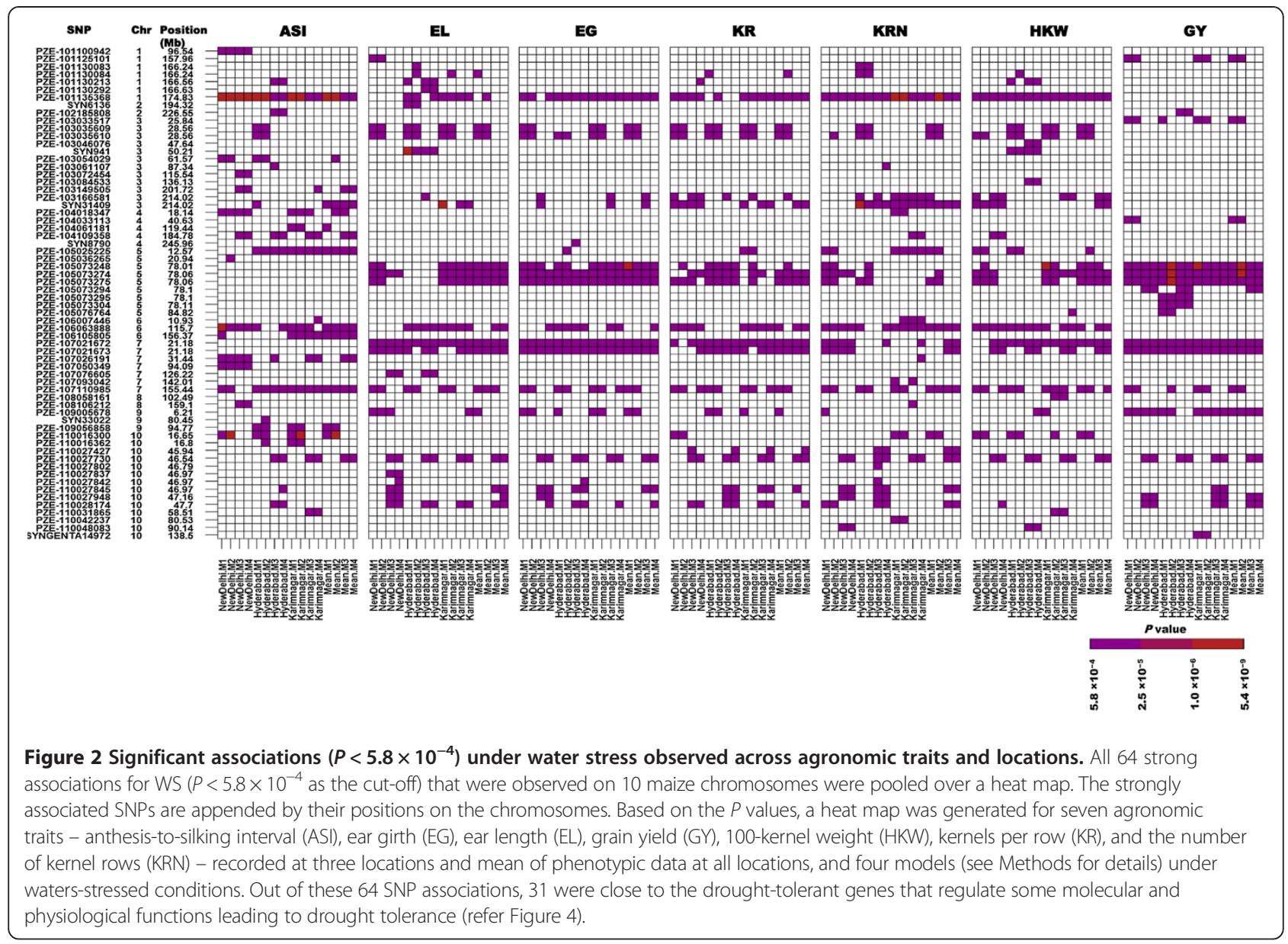

Two SNPs on chromosome 5 (PZE-105025225 and PZE-105076764) mapped close to a gene encoding a $\mathrm{C} 2 \mathrm{H} 2$-type zinc finger $(\mathrm{C} 2 \mathrm{H} 2)$ transcription factor were independently associated for WS-ASI and -GY when measured at Hyderabad. The first SNP (PZE-105025225), $31 \mathrm{~kb}$ from $\mathrm{C} 2 \mathrm{H} 2$, showed a strong association $\left(P=2.4 \times 10^{-5}\right)$ for WS-ASI. The second SNP (PZE-105076764), $7 \mathrm{~kb}$ from $\mathrm{C} 2 \mathrm{H} 2$, was associated with a strong signal $\left(P=1.2 \times 10^{-5}\right)$ for WS-GY [Additional file 3: Table S3], which was non-significant under WW. Also, SNPs mapped on chromosomes 2 (PZE-102185808) and 7 (PZE-107110985) were associated with both WS-ASI and -GY (Figure 3a, b) where the association of the first locus with ASI was more significant in WS than that in non-significant signals observed under WW at Hyderabad. The first locus was mapped near the genes encoding MYB, a bZIP transcription factor, and a zinc finger-homeodomain (ZF-HD) transcription factor, and the second was near bHLH [Additional file 3: Table S3].

For WS-HKW, four strong GWA signals were observed on different chromosomes close to the stress-related genes. The first two loci (PZE-101135368 on chromosome 1 and PZE-105025225 on chromosome 5) were observed $12 \mathrm{~kb}$ and $20 \mathrm{~kb}$ from NF-YA respectively [Additional file 3 : Table S3]. The third locus (PZE-106063888 on chromosome 6), also showing a significant association for WS-HKW $\left(P=6.4 \times 10^{-5}\right)$, was mapped close to one gene, namely vacuolar proton ATPase pump (V-type $H^{+}$pump), and two transcription factors, namely NAC and calmodulin-binding transcription activator (CAMTA) [Additional file 3: Table S3]. The fourth locus (PZE-103035609 mapped on chromosome 3) was close to a universal stress protein [Additional file 3: Table S3].

\section{Discussion}

Metabolic mechanisms common to WS and WW conditions

Strong GWA signals were detected near the genes responsible for drought tolerance as well as for plant metabolism across both WS and WW environments. This commonality highlights the importance of genes that are responsible for maintaining yield and were associated with yield-related traits under both the environments. Two SNPs (PZE-107021672 and PZE-107021673) on chromosome 7 were strongly associated with GY for both WS (Figure 2) and WW data sets [Additional file 4: 

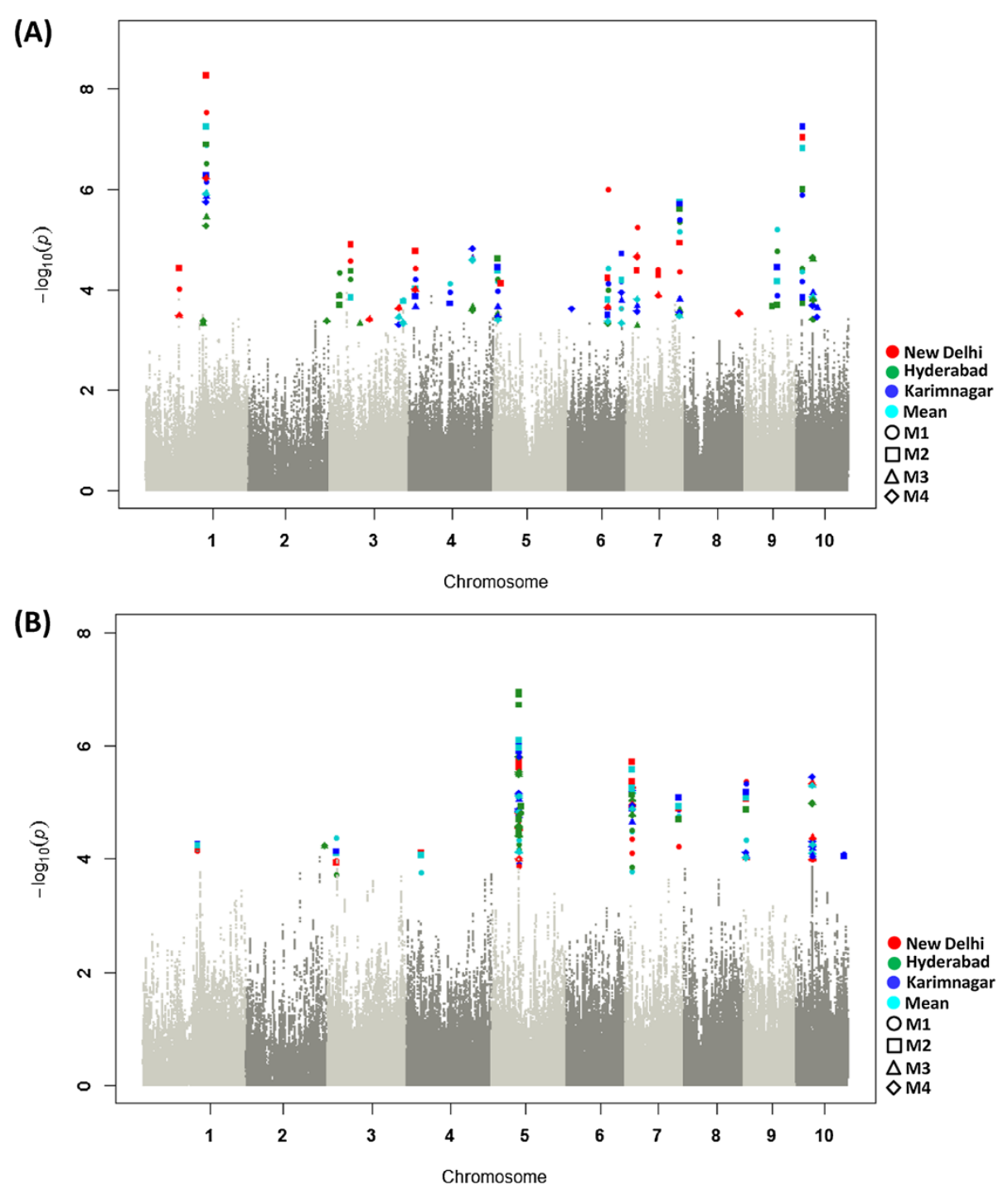

Figure 3 SNPs significantly associated for anthesis-to-silking interval and grain yield across all locations and models under water stress. All $\log _{10} P$ values (<-4.0) observed for a data set were pooled over a GWA plot. (A) Six FDR-corrected associations on 5 chromosomes were observed for WS-ASI specific to locations and models. PZE-101135368 on chromosome 1 demonstrated strong signals at all locations and models, the most significant $\left(P=5.4 \times 10^{-9}\right)$ bring for the data set New Delhi-M2. (B) Eight FDR-corrected associations were observed for WS-GY. Four of these were mapped on chromosome 5, with the PZE-105073248 and PZE-105073275 SNPs showing strong association across all data sets.

Figure S1, Additional file 5: Figure S2, Additional file 6: Figure S3, and Additional file 7: Figure S4]. These SNPs showed a drought-tolerant ERF transcription factor in their vicinity, which promotes ABA-dependent stomatal closure under WS [17] (Figure 4). Apart from this drought-tolerant gene, these SNPs also included two more genes encoding MADS-box and glycerol-3phosphate dehydrogenase (G3PDH), which regulate generalized functions in plants. During root development, the MADS-box gene regulates auxin transport [18], cell proliferation [19], the transition to flowering and flower development [19]. G3PDH is part of the mitochondrial glycerol-3-phosphate shuttle system that is induced at different stages of plant development when the demand for redox adjustment is high [20]. G3PDH is also one of the enzymes involved in glycerol metabolism, where the role of glycerol in regulating root development through multiple pathways in Arabidopsis has been studied recently [21].

A strong GWA signal (PZE-101135368) for ASI and KRN was identified for both WS [Additional file 3: Table S3] and WW across all data sets although the $P$ value varied with the data set [Additional file 4: Figure S1, Additional file 5: Figure S2, Additional file 6: Figure S3, and Additional file 7: Figure S4]. This association pointed to a drought-tolerant NF-YA transcription 


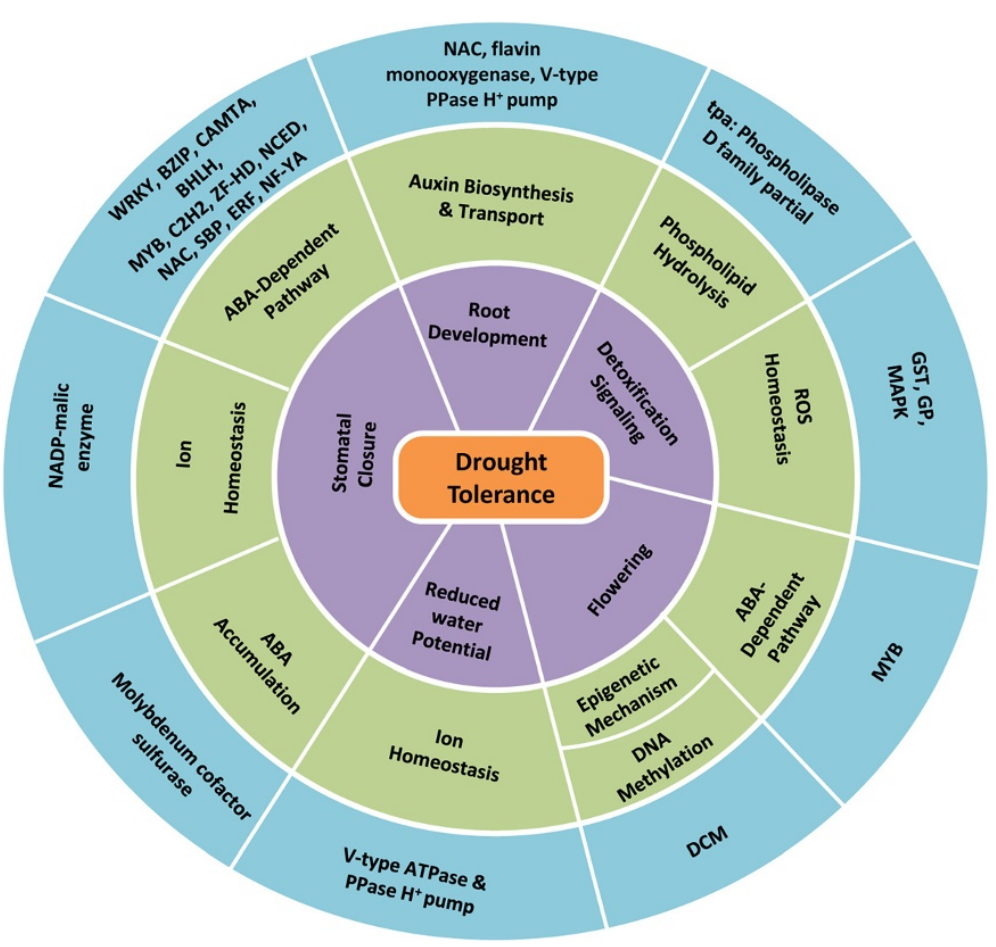

Figure 4 Functional roles of candidate genes associated with drought tolerance. The outermost circle represents the drought-tolerant genes mapped in the present study; the middle circle shows the molecular mechanisms involved in drought tolerance; and the inner circle describes the functional role of the GWA candidate genes that directly lead to drought tolerance.

factor $12 \mathrm{~kb}$ from the SNP. Under WS, NF-YA promotes ABA-dependent stomatal closure, which contributes to drought tolerance [22] (Figure 4). NF-YA is also important to several other functions including male gametogenesis, embryogenesis, and seed development under WW conditions [23]. The consistency of these GWA signals shows that NF-YA is important to high yields irrespective of whether if water is scarce or abundant.

Another strong GWA signal (PZE-107110985) was that for ASI, GY, and KR across both environments and at all locations. $B H L H$, a drought-tolerant gene, was mapped close to this SNP, and has been known to interact with two other two drought-tolerant genes, $b Z I P$ and $M Y B$, which are responsible for tissue-specific flavonoids production and light responsiveness under normal conditions [24]: bZIP regulates auxin transport [25] and $M Y B$ promotes light signalling transductions including photosynthesis in Arabidopsis thaliana. The importance of these genes under WS was noticed from the specific GWA signals for WS that were mapped close to three drought-tolerant gene families: PZE-103061107 near $M Y B, P Z E-104061181$ and SYNGENTA14972 near $b H L H$, and PZE-104109358 near bZIP. Apart from drought tolerance, these genes can also interact strongly to regulate other generalized functions, as can be inferred from the GWA signals mapped across both the environments.

\section{Mechanisms of drought tolerance}

Strong GWA signals were recorded near the interacting drought-tolerant genes under WS [Additional file 3: Table S3] (Figure 4). These mechanisms, namely stomatal closure, detoxification signalling, root development, reduced water potential, and flowering, are influenced at different physiological and functional levels and enhance drought tolerance (Figure 4).

\section{Stomatal closure}

A genomic region encoding two neighbouring SNPs 253 bp apart (PZE-105073274 and PZE-105073275) in high linkage disequilibrium (LD) $\left(r^{2}=1\right)$ [26] was seen on chromosome 5, near two CAMTA regulating factors, the MYB and SBP (Figure 5). These SNPs showed significant $P$ values for several traits including GY, EG, EL, and KR under WS [Additional file 3: Table S3]. The extended regions with strong LD exhibited a steep selection and genetic drift [27] of those traits in the population. CAMTA was also identified near a locus mapped on chromosome 6 that was strongly associated with WS-ASI, -EG, -EL, -KRN, -KR, and -HKW [Additional file 3: Table S3]. CAMTA (at $115 \mathrm{Mb}$ on chromosome 6), MYB, and SBP (at $77.9 \mathrm{Mb}$ on chromosome 5) contributed to stomatal closure, which promotes drought tolerance, presumably through strong 

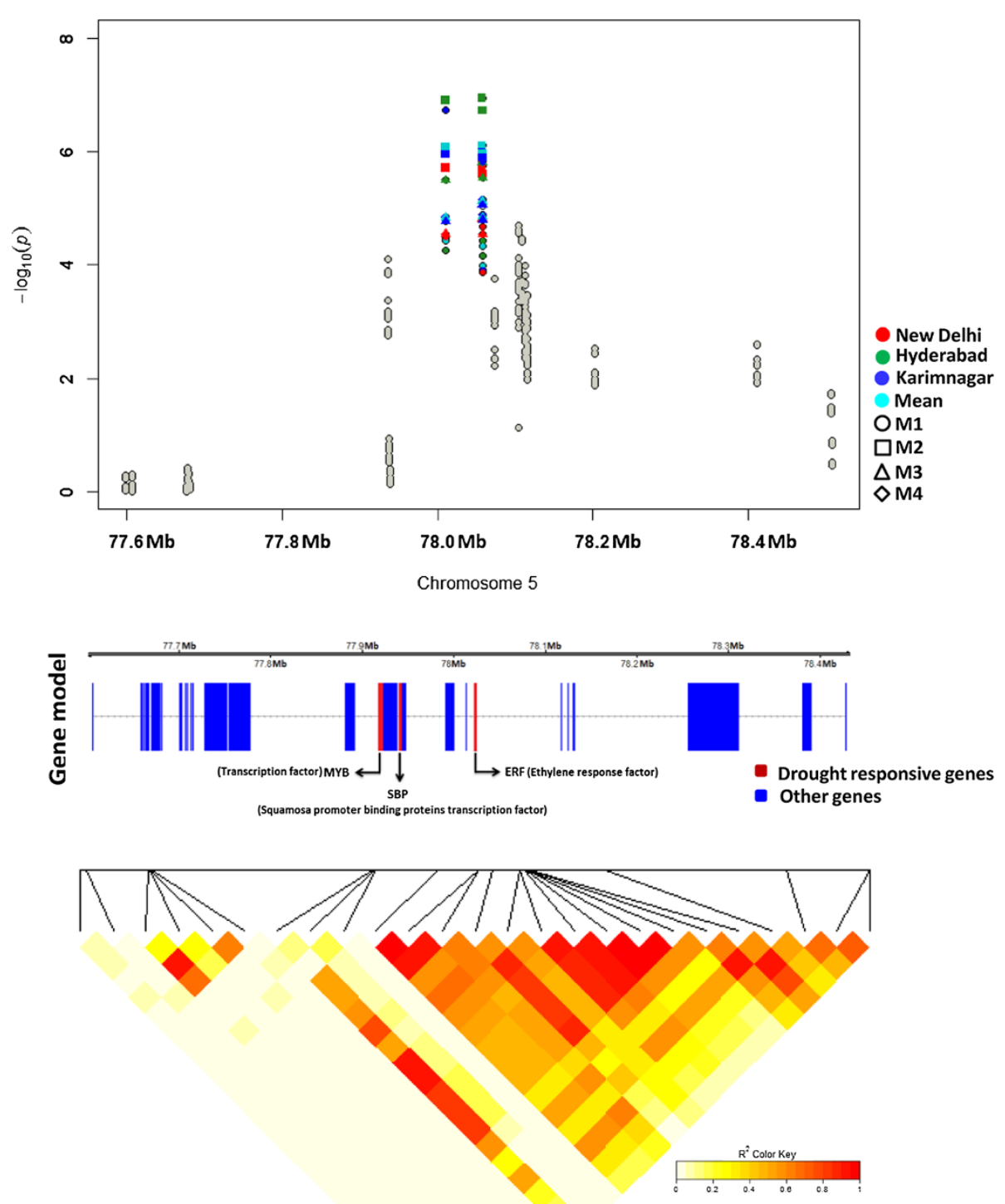

Figure 5 Regional association plot of $1 \mathrm{Mb}$ window on chromosome 5 showing SNPs significantly associated for grain yield under water stress across all locations and models. The genomic region included three neighbouring SNPs (PZE-105073248, PZE-105073274, and PZE-105073275) that were strongly associated with grain yield. PZE-105073274 and PZE-105073275 (78.05 Mb; 253-bp apart) in high linkage disequilibrium $\left(r^{2}=1\right)$ showed FDR-corrected associations specific to locations and models. All three SNPs were observed close to MYB and SBP: $84 \mathrm{~kb}, 130.8 \mathrm{~kb}$, and $131.1 \mathrm{~kb}$ from MYB and $65 \mathrm{~kb}, 111.6 \mathrm{~kb}$, and $111.8 \mathrm{~kb}$ from SBP for PZE-105073248, PZE-105073274, and PZE-105073275 respectively.

inter-chromosomal LD. Other CAMTA-regulating factors such as WRKY and bZIP were mapped near an SNP (PZE-104109358) on chromosome 4 that was strongly associated with WS-ASI, -KRN, and -HKW [Additional file 3: Table S3].

An SNP cluster on chromosome 5 with a significant association for WS-GY and -HKW included the genes encoding $\mathrm{C} 2 \mathrm{H} 2$ and MYB [Additional file 3: Table S3]. Although the function of $\mathrm{C} 2 \mathrm{H} 2$ in maize is not clear, a $\mathrm{C} 2 \mathrm{H} 2$-type zinc finger was shown to play a defensive role in oxidative stress generated under different forms of environmental stress in Arabidopsis [28]. Moreover, a gene of the $M Y B$ family is known to integrate ABA and auxin signalling under WS [29].

In the present study, two SNPs were identified, one on chromosome 3 and one on chromosome 4, with strong associations for WS-ASI and WS-KRN [Additional file 3: Table S3] (Figure 2). The SNP on chromosome 3 encompassed the gene encoding 9-cis-epoxycarotenoid dioxygenase (NCED) whereas the one on chromosome 4 was localized at a point close to a molybdenum cofactor sulfurase encoding gene [Additional file 3: Table S3]. Because 
of possible inter-chromosomal LD, this region may contribute to ABA-dependent stomatal closure. This defensive mechanism can be explained by an integrative ABA synthesis, accumulation [30], and signalling in guard cells [31]. We also identified two SNPs, one on chromosome 3 and another on chromosome 10, close to an NADP-malic enzyme encoding gene [Additional file 3: Table S3], with strong $P$ values for WS-ASI and -GY, respectively (Figure 3a, b). This gene promotes stomatal closure, which induces tolerance during WS, by removing malate from guard cells [32].

\section{Detoxification signalling}

Detoxification signalling is a major response to environmental forms of stress, and WS is known to stimulate oxidative stress in chloroplasts, mitochondria, and peroxisomes [33], thereby leading to the production of reactive oxygen species (ROS) that are sensitive to ABA-signalling pathways [33] and induce programmed cell death in plants [34]. Therefore, removal of free oxygen radicals may represent the primary defence mechanism in drought tolerance. Glutathione-S-transferase (GST) and glutathione peroxidase (GP), which are responsible for ROS homeostasis, change turgor pressure through an ABA-mediated pathway $[35,36]$ in order to sustain the plant under drought. These genes were observed to be strongly associated with WS-GY, - HKW, and -KRN [Additional file 3: Table S3], thereby highlighting the role of ROS homeostasis under WS (Figure 4).

Phospholipid hydrolysis is also known to play a role in detoxification signalling under WS $[37,38]$. In the present study, a genomic region (chromosome 3) encoding phospholipase D (PLD) displayed a strong and WS-specific association signal for GY [Additional file 3: Table S3]. PLD is a signalling messenger that facilitates stomatal closure, cell viability, and enhanced root growth during stress [38].

\section{Root development}

Under WS, auxin signalling results in a deeper root system, which increases water use efficiency $[39,40]$. We noticed two strong association signals in the genomic regions that included the genes for auxin transport (NAC) and auxin biosynthesis (flavin monooxygenase) on chromosomes 6 and 10 respectively [Additional file 3: Table S3]. These regions showed high $P$ values for WS-ASI, -KR, and -HKW, suggesting that maintenance of the root system was in fact associated with flowering and seed development stages.

\section{Reduced water potential}

Vacuolar proton pumps reduce water potential under WS: their expression in the root system enhances waterabsorbing efficiency [41]. The strong signal of the vacuolar proton pyrophosphatase pump (V-type PPase $\mathrm{H}^{+}$pump) for
WS-KRN and that of the V-type ATPase $H^{+}$pump for WS-ASI, -EG, -HKW, -EL, -KRN, and -KR [Additional file 3: Table S3] establish a proton electrochemical gradient across the vacuolar membrane [41,42], thereby enhancing ion homeostasis, which is responsible for maintaining osmotic balance and in turn, coping with drought (Figure 4).

\section{Flowering}

Drought tolerance also involves changes in flowering through epigenetic mechanisms and the ABA-dependent pathway - an observation borne out by the present study (Figure 4) -and DNA cytosine methylation and histone modifications (ABA levels are regulated by histone) are innate to epigenomes $[43,44]$. Our study identified 15 significant SNPs [Additional file 3: Table S3] close to genes encoding DNA-cytosine-5-methyltransferase (DCM) and MYB transcription factor that are involved in epigenetic mechanisms and ABA signalling pathways under WS, thereby pointing to their role in drought tolerance.

\section{Functional relationship of stress-responsive genes}

Interactions of several functional mechanisms associated with drought tolerance are discussed below. Epigenetic changes such as DNA methylation respond differently at tissue and developmental levels. In a study of the association of methylation-specific sites with drought tolerance, methylation was reduced in response to water stress at the tillering stage whereas drought-induced DNA methylation and demethylation were higher at the tillering stage than at the booting and heading stages [45]. Epigenetic changes in $M Y B$, an ABA-signalling transcription factor, have been found to be specific to tissues including pericarp and cob in maize [46]. These epigenetic changes include sitespecific methylation patterns, which have been studied in ABA-signalling genes including the $M Y B$ gene family, where stomatal development genes such as MYB44 are demethylated and expressed in response to WS [47]. MYB proteins also promote stamen development and ensure adaptation to WS [48]. Therefore the epigenetic changes in ABA-signalling genes can possibly lead to changes in flowering under WS (Figure 4).

ROS are produced in an ABA-signalling pathway in which two ABA-signalling transcription factors $\mathrm{C} 2 \mathrm{H} 2$ and MAPK are negatively regulated by ERF [49], which is repressed under WS [50]. ROS are scavenged by a detoxification signalling process [37], in which PLD is reported to influence ABA responses and opening and closing of stomata through bifurcating pathways [51]. When PLD activity was studied under water stress, three ABA-signalling genes, namely $M Y B, N A C$ (PZE-108058161; chromosome 8), and $b H L H$, showed differential regulation in both wild-type and antisensePLD Arabidopsis [52]. Therefore, networking among ABA 
and PLD signals is one of the important defensive mechanisms against WS. This suggests that the ROS generated by ABA signalling are reduced by the interplay between ethylene signalling, ABA signalling, and detoxification signalling under WS.

Guard cell signal transductions regulate $\mathrm{CO}_{2}$ influx for photosynthesis as well as water loss through stomatal closure under WS [31,32]: ABA signalling promotes the closing of stomata, which reduces $\mathrm{CO}_{2}$ intake in guard cells and thereby decreases the rate of photosynthesis. To maintain the rate of photosynthesis under WS, plants adopt an alternative pathway for delivering $\mathrm{CO}_{2}$. This alternative pathway includes malate degradation, catalyzed by a respiratory enzyme, NADP-malic enzyme, which releases $\mathrm{CO}_{2}$ as a secondary source for $\mathrm{CO}_{2}$ influx under WS [32].

$\mathrm{ABA}$ is a hormone released in response to various forms of abiotic stress and regulates tolerance through a network of interconnected genes. The mechanisms of drought tolerance are stimulated by both ABA-dependent and ABA-independent pathways: $\mathrm{C} 2 \mathrm{H} 2$, for instance, is part of an ABA-dependent pathway as well as that of an ABAindependent pathway. $\mathrm{C} 2 \mathrm{H} 2$ zinc finger proteins with ERF-associated amphiphilic repression (EAR) motifs have been reported to be responsive to ABA [53] and to WS [54].These findings suggest that $A B A$ and ethylene interact under drought and enhance tolerance by promoting stomatal closure. Under WS, $\mathrm{C} 2 \mathrm{H} 2$ acts as a transcriptional regulator through an $\mathrm{ABA}$-independent pathway [55]: $\mathrm{ABA}$-independent $\mathrm{C} 2 \mathrm{H} 2$ triggers the genes that are related to $\mathrm{H}_{2} \mathrm{O}_{2}$ homeostasis and thus decreases the amount of $\mathrm{H}_{2} \mathrm{O}_{2}$ in guard cells [56]. However, stomatal closure as part of the drought response is promoted by ABA and $\mathrm{H}_{2} \mathrm{O}_{2}$ accumulation in guard cells [56].

The electrochemical gradient generated by the proton pump (V-type PPase $H^{+}$pump mapped near PZE110027802; chromosome 10) promotes the secondary active transport of sugar molecules (hexokinase 3 mapped near PZE-108106212; chromosome 8) into the vacuole. This helps the plant in maintaining its internal water balance [57]. In addition, the proton pump has also been reported in roots [42], which suggest that this pump regulates water balance in roots and thus contributes to drought tolerance. It is well understood that the water balance in plants is interrelated to root development, where the auxin-inducible genes that promote root development are regulated by the $\mathrm{C} 2 \mathrm{H} 2$ [58]. This suggests that the integration of root development and $\mathrm{ABA}$ signalling molecule $\mathrm{C} 2 \mathrm{H} 2$ allows the maintenance of an electrochemical gradient in the plant.

\section{Conclusions}

Genome-wide association analysis, using multiple locations and two models, identified SNPs from agronomic traits linked with the genes directly or indirectly associated with drought tolerance in subtropical maize lines. These genes uncovered physiological responses and molecular mechanisms related to drought tolerance. Genes governing several functional traits were identified, including stomatal closure, reduced water potential, root development, signalling pathways, and flowering. These genes interact extensively to help the plant cope with drought. SNPs and their functional association with several drought-responsive genes will be useful in elucidating the mechanism of drought tolerance in subtropical maize.

\section{Methods}

\section{Genetic material}

Our experiment was based on a panel of 240 elite inbred lines of subtropical maize [26] from several Indian breeding programmes and also included genotypes from CIMMYT, Mexico adapted to subtropical climates. The lines were separated into three groups, namely early, medium, and late, based on the number of days to flowering.

\section{Phenotyping}

Field experiments were laid out in an alpha-lattice design at three locations: IARI, New Delhi $\left(28^{\circ} \mathrm{N} 77^{\circ} \mathrm{E} ; 229 \mathrm{Amsl}\right)$; Maize Research Centre, Acharya N G Ranga Agricultural University, Hyderabad $\left(17^{\circ} \mathrm{N} 78^{\circ} \mathrm{E} ; 536 \mathrm{Amsl}\right)$, and ARS, Acharya N G Ranga Agricultural University, Karimnagar $\left(18^{\circ} \mathrm{N} 79^{\circ} \mathrm{E} ; 264\right.$ Amsl) during the post-rainy seasons of 2010/11 and 2011/12. The design comprised 16 incomplete blocks, each made up of 15 plots with three replicates. Sowing was staggered to ensure that all the lines - whether early, medium, or late - are exposed to stress at the same stage of their growth. WS was induced at the flowering stage and continued until grain filling by withholding irrigation, which was resumed in all the maturity groups at the end of the stress treatment. The following observations were recorded for all the 240 lines: anthesis-to-silking interval (ASI, in days), ear girth (EG, in centimetres), ear length (EL, in centimetres), the number of kernels per row (KR), the number of kernel rows (KRN), 100-kernel weight (HKW, in grams), and grain yield (GY, kilograms per plot).

\section{SNP genotyping}

All the 240 genotypes were genotyped with Infinium Maize SNP50 BeadChip (Illumina, San Diego, California, USA) containing $56110 \mathrm{SNPs}$ published earlier by Nepolean et al [26]. A set of 29619 high-quality SNPs [26] was used for association analysis.

\section{Data analysis \\ Field data}

Mixed model analysis using restricted maximum likelihood approach was performed to estimate the best linear unbiased predictors (BLUPs) of genotypes at each location. 
Plot-level data from each location were analyzed using the following linear mixed-effects model:

$$
y_{i j k}=\mu+r_{i}+r(b)_{i j}+g_{k}+\varepsilon_{i j k}
$$

where $y_{i j k}$ is the observed value of the $k^{\text {th }}$ genotype in the $j^{\text {th }}$ incomplete block within the $i^{\text {th }}$ replication, $\mu$ is the grand mean, $r_{i}$ the main effect of the $i^{\text {th }}$ replication, $r(b)_{i j}$ the nested effect of the $j^{\text {th }}$ incomplete block in $i^{\text {th }}$ replication, $g_{k}$ is the main effect of the $k^{\text {th }}$ genotype, and $\varepsilon_{i j k}$ is the error term of each $y_{i j k}$ with $N\left(0, \sigma^{2}\right)$. At each location, the nested blocks and genotype effects were treated as random and replicated as fixed effects. The residual diagnostic plots indicated that the data satisfied the assumption of normality. To understand the effect of genotypes across locations in greater detail, the data across the three locations were analyzed using the model

$$
y_{m i j k}=\mu+l_{m}+l(r)_{i}+l(r b)_{i j}+g_{k}+(g l)_{m k}+\varepsilon_{m i j k}
$$

where $l_{m}$ is the main effect of $m^{\text {th }}$ location and $(g l)_{m k}$ is the interaction effect of $k^{\text {th }}$ genotype in $m^{\text {th }}$ location. Residual variances of individual locations were modelled in combined analysis using a mixed model procedure. BLUPs for the genotypes were also estimated across combined locations. Data were analyzed using a proc mixed procedure of the software package SAS ver. 9.3 for Windows (SAS Institute Inc.) [59].

\section{Genome-wide association analysis}

Two models, $\mathrm{Q}_{10}+\mathrm{K}+$ Admixture from GenABEL and $\mathrm{Q}_{10}+\mathrm{K}+$ Admixture from GAPIT were used to identify significant SNPs from BLUPs. Here, admixture values were obtained from earlier data for the same panel [26]. In GenABEL, a mixed linear model (MLM) was employed with polygenic (maximum likelihood method) [60] and "mmscore" functions [61]. In the present study, the K estimate represents the identity by state (IBS) shared by individuals computing to IBS or kinship matrix generated from GenABEL [60]. The population structure was corrected for the first ten components $\left(\mathrm{Q}_{10}\right)[12,62]$ by implementing the "cmdscale" function in GenABEL [68]. $P$ values with 1 degree of freedom were corrected at 5\% FDR using the "qvaluebh95" function [63] in GenABEL. In GAPIT, GWAS was investigated by the compressed mixed linear model (CMLM) approach with "EMMAx" (Efficient Mixed Model Association-eXpedited) [64], and $P$ values were adjusted at 5\% FDR to determine significant associations [64]. To compute kinship matrix, the VanRaden algorithm [65] was implemented. Admixture and principal component analysis (PCA) were used to correct the population structure.

\section{Availability of supporting data}

The raw SNP data (Submission \# 10.6070/H4BG2KX8) has been submitted to the website: http://www.labarchives.com/.
All the supporting data are included as additional files (Additional files 1, 2, 3, 4, 5, 6, 7, 8, 9, 10, 11).

\section{Additional files}

Additional file 1: Table S1. Agronomic performance of genotypes in well-watered and water-stressed conditions.

Additional file 2: Table S2. Phenotypic correlation coefficients for agronomic traits in well-watered and water-stressed conditions. Significant phenotypic correlations $(p<0.01)$ are marked with an asterisk.

Additional file 3: Table S3. GWA signals observed for seven agronomic traits under water stress. Significant associations $\left(P<5.8 \times 10^{-4}\right.$ as the cut-off) for all agronomic traits were observed at all the three locations (New Delhi, Hyderabad, and Karimnagar) and for the mean of all phenotypic data and four models (M1, M2, M3, and M4) (Refer Methods for details). All FDR-corrected SNP associations are in bold. The drought-tolerant gene annotations (refer Figure 4) are marked with the respective references.

Additional file 4: Figure S1. Manhattan plots showing SNPS significantly associated $\left(\log _{10} P<-4.0\right)$ with seven agronomic traits under well-watered conditions measured across M2 (see Methods for details).

Additional file 5: Figure S2. Manhattan plots showing SNPS significantly associated $\left(\log _{10} P<-4.0\right)$ to seven agronomic traits under well-watered conditions measured across M1 (see Methods for details).

Additional file 6: Figure S3. Manhattan plots showing SNPS significantly associated $\left(\log _{10} P<-4.0\right)$ to seven agronomic traits under well-watered conditions measured across M3 (see Methods for details).

Additional file 7: Figure S4. Manhattan plots showing SNPS significantly associated $\left(\log _{10} P<-4.0\right)$ to seven agronomic traits under well-watered conditions measured across M4 (see Methods for details).

Additional file 8: Figure S5. Manhattan plots showing SNPS significantly associated $\left(\log _{10} P<-4.0\right)$ to seven agronomic traits under water stress measured across M1 (see Methods for details).

Additional file 9: Figure S6. Manhattan plots showing SNPS significantly associated $\left(\log _{10} P<-4.0\right)$ to seven agronomic traits under water stress measured across M3 (see Methods for details).

Additional file 10: Figure S7. Manhattan plots showing SNPS significantly associated $\left(\log _{10} P<-4.0\right)$ to seven agronomic traits under water stress measured across M4 (see Methods for details).

Additional file 11: Figure S8. Manhattan plots showing SNPS significantly associated $\left(\log _{10} P<-4.0\right)$ with seven agronomic traits under water stress measured across M2 (see Methods for details).

\section{Abbreviations}

ABA: Abscisic acid; bHLH: Basic helix-loop-helix; BLUP: Best linear unbiased predictors; CAMTA: Calmodulin binding transcription activator; DCM: DNA-cytosine-5-methyltransferase; ERF: Ethylene responsive factor; FDR: False-discovery-rate; G3PDH: Glycerol-3-phosphate dehydrogenase; GP: Glutathione peroxidase; GST: Glutathione-S-transferase; GWA: Genome-wide association; LD: Linkage disequilibrium; MLM: Mixed linear model; NCED: 9-cis-epoxycarotenoid dioxygenase; NF-YA: Nuclear factor-YA; PLD: Phospholipase D; QTL: Quantitative trait locus; ROS: Reactive oxygen species; SBP: Squamous promoter binding protein; SNP: Single nucleotide polymorphism; ZF-HD: Zinc finger-homeodomain.

\section{Competing interests}

The authors declare that they have no competing interest.

\section{Authors' contributions}

NT and HSG conceived and designed the experiments; KS, SMt, SMn and PMN generated genotyping data; FH, SD, TSR, and SK generated phenotyping data; RS, KA, KS, SMt, SMn, AR, and TS analyzed the data; TM helped with SNP data generation; and NT and KA drafted the paper. HSG coordinated the research. All authors read and approved the final manuscript. 


\section{Acknowledgements}

We thank the breeders from different breeding centres in India and from CIMMYT for providing the seeds, and the National Agricultural Innovation Project (NAIP, Component IV) and the ICAR Network Project on Transgenics in Crop Plants (Maize Functional Genomics Component) for funding the study.

\section{Author details}

'Division of Genetics, Indian Agricultural Research Institute, Pusa, New Delhi 110012, India. ${ }^{2}$ Maize Research Centre, ARI, Acharya N G Ranga Agricultural University, Rajendra Nagar, Hyderabad 500030, India. ${ }^{3}$ Agricultural Research Station, Acharya N G Ranga Agricultural University, Karimnagar 505001, India. ${ }^{4}$ International Crops Research Institute for the Semi-Arid Tropics, Patancheru 502324, India. ${ }^{5}$ National Research Centre on Plant Biotechnology, Pusa, New Delhi 110012, India.

Received: 9 October 2014 Accepted: 16 December 2014 Published: 24 December 2014

\section{References}

1. Schoper JB, Lambert RJ, Vasilas BL: Maize pollen viability and ear receptivity under water and high temperature stress. Crop Sci 1986, 26:1029-1033.

2. Edmeades GO, Bolaños J, Hernandez M, Bello S: Causes for silk delay in lowland tropical maize. Crop Sci 1993, 33:1029-1035.

3. Magorokosho C, Pixley KV, Tongoona P: Selection for drought tolerance in two tropical maize populations. African Crop Sci J 2003, 11:151-161.

4. Russell WA: Genetic improvements of maize yields. Adv Agron 1991, 46:245-298.

5. Bolaños J, Edmeades GO: The importance of the anthesis-silking interval in breeding for drought tolerance in tropical maize. Field Crops Res 1996, 48:65-80.

6. Setter TL, Yan J, Warburton M, Ribaut JM, Xu Y, Sawkins M, Buckler ES, Zhang Z, Gore MA: Genetic association mapping identifies single nucleotide polymorphisms in genes that affect abscisic acid levels in maize floral tissues during drought. J Exp Bot 2011, 62:701-716.

7. Kakumanu A, Ambavaram MM, Klumas C, Krishnan A, Batlang U, Myers E, Grene R, Pereira A: Effects of drought on gene expression in maize reproductive and leaf meristem tissue revealed by RNA-Seq. Plant Physiol 2012, 160:846-867.

8. Pandey N, Ranjan A, Pant P, Tripathi RK, Ateek F, Pandey HP, Patre UV, Sawant SV: CAMTA 1 regulates drought responses in Arabidopsis thaliana. BMC Genomics 2013, 14:216.

9. Lu M, Ying S, Zhang D-F, Shi Y-S, Song Y-C, Wang T-Y, Li Y: A maize stress-responsive NAC transcription factor, ZmSNAC1, confers enhanced tolerance to dehydration in transgenic Arabidopsis. Plant Cell Rep 2012, $31: 1701-1711$

10. Xue Y, Warburton ML, Sawkins M, Zhang X, Setter T, Xu Y, Grudloyma P, Gethi J, Ribaut JM, Li W, Zhang X, Zheng Y, Yan J: Genome-wide association analysis for nine agronomic traits in maize under well-watered and water-stressed conditions. Theor Appl Genet 2013, 10.1007/s00122-013-2158-X.

11. Tian F, Bradbury PJ, Brown PJ, Hung H, Sun Q, Flint-Garcia S, Rocheford TR, McMullen MD, Holland JB, Buckler ES: Genome-wide association study of leaf architecture in the maize nested association mapping population. Nat Genet 2011, 43:159-162.

12. Riedelsheimer C, Lisec J, Czedik-Eysenberg A, Sulpice R, Flis A, Grieder C, Altmann T, Stitt M, Willmitzer L, Melchinger AE: Genome-wide association mapping of leaf metabolic profiles for dissecting complex traits in maize. Proc Natl Acad Sci U S A 2012, 109:8872-8877.

13. Salvi S, Sponza G, Morgante M, Tomes D, Niu X, Fengler KA, Meeley R, Ananiev EV, Svitashev S, Bruggemann E, Li B, Hainey CF, Radovic S, Zaina G, Rafalski JA, Tingey SV, Miao G-H, Phillips RL, Tuberosa R: Conserved noncoding genomic sequences associated with a flowering-time quantitative trait locus in maize. Proc Natl Acad Sci U S A 2007, 104:11376-11381.

14. Li Q, Li L, Yang XH, Warburton ML, Bai GH, Dai JR, Li JS, Yan JB: Relationship, evolutionary fate and function of two maize orthologous genes of rice GW2 associated with kernel size and weight. BMC Plant Biol 2010, 10:143.

15. Manicacci D, Camus-Kulandaivelu L, Fourmann M, Arar C, Barrault S, Rousselet A, Feminias N, Consoli L, Francès L, Méchin V, Murigneux A, Prioul JL, Charcosset A, Damerval C: Epistatic interactions between opaque2 transcriptional activator and its target gene CyPPDK1 control kernel trait variation in maize. Plant Physiol 2009, 150:506-520.

16. Almeida GD, Makumbi D, Magorokosho C, Nair S, Borem A, Ribaut JM, Banziger M, Prasanna BM, Crossa J, Babu R: QTL mapping in three tropical maize populations reveals a set of constitutive and adaptive genomic regions for drought tolerance. Theor Appl Genet 2013, 126:583-600.

17. Cheng MC, Liao PM, Kuo WW, Lin TP: The Arabidopsis ETHYLENE-RESPONSEFACTOR1 regulates abiotic-stress-responsive gene expression by binding to different cis-acting elements in response to different stress signals. Plant Physiol 2013, 162:1566-1582.

18. Garay-Arroyo A, Ortiz-Moreno E, de la Paz SM, Murphy AS, García-Ponce B, Marsch-Martínez N, de Folter S, Corvera-Poiré A, Jaimes-Miranda F, Pacheco-Escobedo MA, Dubrovsky JG, Pelaz S, Álvarez-Buylla ER: The MADS transcription factor XAL2/AGL14 modulates auxin transport during Arabidopsis root development by regulating PIN expression. EMBO J 2013, 32:2884-2895.

19. Tapia-Lopez R, García-Ponce B, Dubrovsky JG, Garay-Arroyo A, Pérez-Ruiz RV Kim SH, Acevedo F, Pélaz S, Alvarez-Buylla ER: An AGAMOUS-related MADS-box gene, XAL1 (AGL12), regulates root meristem cell proliferation and flowering transition in Arabidopsis. Plant Physiol 2008, 146:1 182-1192.

20. Shen W, Wei Y, Dauk M, Tan Y, Taylor DC, Selvaraj G, Zou J: Involvement of a glycerol-3-phosphate dehydrogenase in modulating the NADH/NAD ${ }^{+}$ratio provides evidence of a mitochondrial glycerol-3-phophate shuttle in Arabidopsis. Plant Cell 2006, 18:422-441

21. Hu J, Zhang Y, Wang J, Zhou Y: Glycerol affects root development through regulation of multiple pathways in Arabidopsis. PLoS One 2014, 9:e86269.

22. Li WX, Oono Y, Zhu J, He XJ, Wu JM, lida K, Lu XY, Cui X, Jin H, Zhu JK: The Arabidopsis NFYA5 transcription factor is regulated transcriptionally and post transcriptionally to promote drought resistance. The Plant Cell 2008, 20:2238-2251.

23. Mu J, Tan H, Hong S, Liang Y, Zuo J: Arabidopsis transcription factor genes $\mathrm{NF}-\mathrm{YA} 1,5,6$, and 9 play redundant roles in male gametogenesis, embryogenesis, and seed development. Mol Plant 2012, 6:188-201

24. Hartmann U, Sagasser M, Mehrtens F, Stracke R, Weisshaar B: Differential combinatorial interactions of cis-acting elements recognized by R2R3-MYB, BZIP, and BHLH factors control light-responsive and tissue-specific activation of phenylpropanoid biosynthesis genes. Plant Mol Biol 2005, 57:155-171.

25. Brown DE, Rashotte AM, Murphy AS, Normanly J, Tague BW, Peer WA, Taiz L, Muday GK: Flavonoids act as negative regulators of auxin transport in vivo in Arabidopsis. Plant Physiol 2001, 126:524-535.

26. Nepolean T, Hossain F, Shiriga K, Mittal S, Arora K, Rathore A, Mohan S, Shah T, Sharma R, Namratha PM, Amitha Mithra SV, Mohaptara T, Gupta HS: Unraveling the genetic architecture of subtropical maize (Zea mays $\mathrm{L}$.) lines and their utility in breeding programs. BMC Genomics 2013, 14:877.

27. Barton NH: Genetic hitchhiking. Philos Trans R Soc Lond B Biol Sci 2000, 355:1553-1562

28. Davletova S, Schlauch K, Coutu J, Mittler R: The zinc-finger protein zat12 plays a central role in reactive oxygen and abiotic stress signaling in Arabidopsis. Plant Physiol 2005, 139:847-856.

29. Seo PJ, Xiang F, Qiao M, Park JY, Lee YN, Kim SG, Lee YH, Park WJ, Park CM The MYB96 transcription factor mediates abscisic acid signaling during drought stress response in Arabidopsis. Plant Physiol 2009, 151:275-289.

30. Lu Y, Li Y, Zhang J, Xiao Y, Yue Y, Duan L, Zhang M, Li Z: Overexpression of Arabidopsis molybdenum cofactor sulfurase gene confers drought tolerance in maize (Zea mays L.). PLoS One 2013, 8:e52126.

31. Schroeder Jl, Kwak JM, Allen GJ: Guard cell abscisic acid signalling and engineering drought hardiness in plants. Nature 2001, 410:327-330.

32. Laporte MM, Shen B, Tarczynski MC: Engineering for drought avoidance: expression of maize NADP-malic enzyme in tobacco results in altered stomatal function. J Exp Bot 2002, 53:699-705.

33. Cruz de Carvalho MH: Drought stress and reactive oxygen species. Plant Signal Behav 2008, 3:156-165.

34. Van Breusegem F, Dat JF: Reactive oxygen species in plant cell death. Plant Physiol 2006, 141:384-390.

35. Miao Y, Lv D, Wang P, Wang XC, Chen J, Miao C, Song CP: An Arabidopsis glutathione peroxidase functions as both a redox transducer and a scavenger in abscisic acid and drought stress responses. The Plant Cell 2006, 18:2749-2766.

36. Chen JH, Jiang HW, Hsieh EJ, Chen HY, Chien CT, Hsieh HL, Lin TP: Drought and salt stress tolerance of an Arabidopsis glutathione S-transferase U17 
knockout mutant are attributed to the combined effect of glutathione and abscisic acid. Plant Physiol 2012, 158:340-351.

37. Zhu JK: Salt and drought signal transduction in plants. Annu Rev Plant Biol 2002, 53:247-273.

38. Hong $Y$, Zhang W, Wang X: Phospholipase D and phosphatidic acid signalling in plant response to drought and salinity. Plant Cell Environ 2010, 33:627-635.

39. Hund A, Ruta N, Liedgens M: Rooting depth and water use efficiency of tropical maize inbred lines, differing in drought tolerance. Plant Soil 2009, 318:311-325.

40. Overvoorde P, Fukaki H, Beeckman T: Auxin control of root Development. Cold Spring Harb Perspect Biol 2010, 2:a001537.

41. Pasapula V, Shen G, Kuppu S, Paez-Valencia J, Mendoza M, Hou P, Chen J, Qiu X, Zhu L, Zhang X, Auld D, Blumwald E, Zhang H, Gaxiola R, Payton P: Expression of an Arabidopsis vacuolar $\mathrm{H}+$-pyrophosphatase gene (AVP1) in cotton improves drought- and salt tolerance and increases fibre yield in the field conditions. Plant Biotechnol J 2011, 9:88-99.

42. Li B, Wei A, Song C, Li N, Zhang J: Heterologous expression of the TsVP gene improves the drought resistance of maize. Plant Biotechnol J 2008, 6:146-159.

43. Ratcliffe OJ, Riechmann JL: Arabidopsis transcription factors and the regulation of flowering time: a genomic perspective. Curr Issues Mol Biol 2002, 4:77-91.

44. Chinnusamy V, Gong Z, Zhu JK: Abscisic acid-mediated epigenetic processes in plant development and stress responses. J Integr Plant Biol 2008, 50:1187-1195.

45. Wang WS, Pan YJ, Zhao XQ, Dwivedi D, Zhu LH, Ali J, Fu BY, Li ZK: Drought-induced site-specific DNA methylation and its association with drought tolerance in rice (Oryza sativa L.). J Exp Bot 2010, 62:1951-1960.

46. Cocciolone SM, Chopra S, Flint-Garcia SA, McMullen MD, Peterson T: Tissue-specific patterns of a maize Myb transcription factor are epigenetically regulated. The Plant J 2001, 27:467-478.

47. Colaneri AC, Jones AM: Genome-wide quantitative identification of DNA differentially methylated sites in Arabidopsis seedlings growing at different water potential. PLoS One 2013, 8:e59878.

48. Su Z, Ma X, Guo H, Sukiran NL, Guo B, Assmann SM, Ma H: Flower development under drought stress: morphological and transcriptomic analyses reveal acute responses and long-term acclimation in Arabidopsis. Plant Cell 2013, 25:3785-3807.

49. Sewelam N, Kazan K, Thomas-Hall SR, Kidd BN, Manners JM, Schenk PM: Ethylene response factor 6 is a regulator of reactive oxygen species signaling in Arabidopsis. PLoS One 2013, 8:e70289.

50. Joo J, Choi HJ, Lee YH, Kim YK, Song SI: A transcriptional repressor of the ERF family confers drought tolerance to rice and regulates genes preferentially located on chromosome 11. Planta 2013, 238:155-170.

51. Mishra G, Zhang W, Deng F, Zhao J, Wang X: A bifurcating pathway directs abscisic acid effects on stomatal closure and opening in Arabidopsis. Science 2006, 312:264-266.

52. Mane S, Vasquez-Robinet C, Sioson A, Heath L, Grene R: Early PLDa-mediated events in response to progressive drought stress in Arabidopsis: a transcriptome analysis. J Exp Bot 2007, 58:241-252.

53. Kam J, Gresshoff P, Shorter R, Xue GP: The Q-type $\mathrm{C}_{2} \mathrm{H}_{2}$ zinc finger subfamily of transcription factors in Triticum aestivum is predominantly expressed in roots and enriched with members containing an EAR repressor motif and responsive to drought stress. Plant Mol Biol 2008 , 67:305-322.

54. Sakamoto H, Maruyama K, Sakuma Y, Meshi T, Iwabuchi M, Shinozaki K, Yamaguchi-Shinozaki K: Arabidopsis Cys2/His2-type zinc-finger proteins function as transcription repressors under drought, cold, and high-salinity stress conditions. Plant Physiol 2004, 136:2734-2746.

55. Huang J, Wang JF, Wang QH, Zhang HS: Identification of a rice zinc finger protein whose expression is transiently induced by drought, cold but not by salinity and abscisic acid. DNA Seq 2005, 16:130-136.

56. Huang XY, Chao DY, Gao JP, Zhu MZ, Shi M, Lin HX: A previously unknown zinc finger protein, DST, regulates drought and salt tolerance in rice via stomatal aperture control. Genes Dev 2009, 23:1805-1817.

57. Leigh RA: In The Plant Vacuole. Volume 25. Edited by Leigh RA, Sanders D. San Diego: Academic Press; 1997:171-194.

58. Kodaira KS, Qin F, Tran LS, Maruyama K, Kidokoro S, Fujita Y, Shinozaki K, Yamaguchi-Shinozaki K: Arabidopsis Cys2/His2 zinc-finger proteins AZF1 and AZF2 negatively regulate abscisic acid-repressive and auxin-inducible genes under abiotic stress conditions. Plant Physiol 2011, 157:742-756

59. Base SAS 9.3 Procedures Guide. Cary: SAS Institute Inc:; 2011.

60. Aulchenko YS, Ripke S, Isaacs A, van Duijn CM: GenABEL: an R library for genome-wide association analysis. Bioinformatics 2007, 23:1294-1296.

61. Chen WM, Abecasis GR: Family-based association tests for genomewide association scans. Am J Hum Genet 2007, 81:913-926.

62. Yu J, Pressoir G, Briggs WH, Vroh Bi I, Yamasaki M, Doebley JF, McMullen MD, Gaut BS, Nielsen DM, Holland JB, Kresovich S, Buckler ES: A unified mixed-model method for association mapping that accounts for multiple levels of relatedness. Nat Genet 2006, 38:203-208.

63. Benjamini $Y$, Hochberg $Y$ : Controlling the false discovery rate: a practical and powerful approach to multiple testing. J R Stat Soc B 1995, 57:289-300

64. Lipka AE, Tian F, Wang Q, Peiffer J, Li M, Bradbury PJ, Gore M, Buckler ES, Zhang Z: GAPIT: genome association and prediction integrated tool. Bioinformatics 2012, 28:2397-2399.

65. VanRaden PM: Efficient methods to compute genomic predictions. J Dairy Sci 2008, 91:4414-4423.

doi:10.1186/1471-2164-15-1182

Cite this article as: Thirunavukkarasu et al:: Functional mechanisms of drought tolerance in subtropical maize (Zea mays L.) identified using genome-wide association mapping. BMC Genomics 2014 15:1182.

\section{Submit your next manuscript to BioMed Central and take full advantage of:}

- Convenient online submission

- Thorough peer review

- No space constraints or color figure charges

- Immediate publication on acceptance

- Inclusion in PubMed, CAS, Scopus and Google Scholar

- Research which is freely available for redistribution
() Biomed Central 